\title{
BOUNDED RATIONALITY, MONETARY POLICY, AND MACROECONOMIC STABILITY
}

\author{
FRANCISCO ILABACA, GRETA MEGGIORINI, AND FABIO MILANI
}

\begin{abstract}
This paper estimates a Behavioral New Keynesian model to revisit the evidence that passive US monetary policy in the pre-1979 sample led to indeterminate equilibria and sunspot-driven fluctuations, while active policy after 1982, by satisfying the Taylor principle, was instrumental in restoring macroeconomic stability.

The model assumes "cognitive discounting", i.e., consumers and firms pay less attention to variables further into the future. We estimate the model allowing for both determinacy and indeterminacy.

The empirical results show that determinacy is preferred both before and after 1979. Even if monetary policy is found to react only mildly to inflation pre-Volcker, the substantial degrees of bounded rationality that we estimate prevent the economy from falling into indeterminacy.
\end{abstract}

Keywords: Behavioral New Keynesian Model, Cognitive Discounting, Estimation Under Determinacy and Indeterminacy, Taylor Principle, Active vs Passive Monetary Policy. JEL classification: E31, E32, E52, E58, E70.

Date: June, 2019.

Francisco Ilabaca: Department of Economics, 3151 Social Science Plaza, University of California, Irvine, CA 92697-5100, U.S.A. Email: filabaca@uci.edu. Homepage: https://sites.google.com/view/filabaca.

Greta Meggiorini: Department of Economics, 3151 Social Science Plaza, University of California, Irvine, CA 92697-5100, U.S.A. Email: gmeggior@uci.edu.

Fabio Milani (corresponding author): Department of Economics, 3151 Social Science Plaza, University of California, Irvine, CA 92697-5100, U.S.A. Email: fmilani@uci.edu. Homepage: http://www.socsci.uci.edu/ fmilani. 


\section{InTRODUCTION}

One of the most well-known and influential results in New Keynesian macroeconomics is the evidence that US monetary policy has changed from passive (i.e., one that fails to raise interest rates aggressively enough in response to inflation) in the pre-1979 period, to active after 1979 (Clarida, Galí, and Gertler, 2000, Lubik and Schorfheide, 2004). A failure by the central bank to conduct monetary policy in a way that satisfies the Taylor principle is conducive to indeterminacy and macroeconomic instability in a benchmark New Keynesian model. A regime switch in monetary policy is typically proposed as an explanation for the high volatility and high inflation outcomes in the 1970s, and the more favorable macroeconomic outcomes in the 1980s and 1990s. ${ }^{1}$

The result of a switch from passive to active monetary policy has been obtained under the assumption that economic agents have rational expectations, but also complete attention, and full information and understanding about the future. The rational expectations hypothesis, however, implies a very large weight given to expectations far into the future, with only minimal discounting. Since determinacy or indeterminacy of the equilibrium is a property of the whole model, a failure to recognize and explicitly model deviations from benchmark models of expectations formation may skew the results.

This paper adds behavioral elements to the New Keynesian model, by introducing "cognitive discounting", or myopia, as in Gabaix (2018). The model is based on microfoundations developed previously in Gabaix $(2014,2016)$. Agents still maximize their utility, but they form expectations with perceived laws of motion that are subject to an attenuated cognitive discount factor, i.e., they pay more limited attention to events that are more distant into the future.

We estimate the Behavioral New Keynesian model by allowing for parameter values that fall both in the determinacy or indeterminacy regions. The challenges related to the solution and estimation of the model in the region of indeterminacy are solved by using the techniques proposed by Bianchi and Nicoló (2019). We estimate the behavioral parameters, such as the extent of cognitive discounting, along with the structural, and Taylor rule parameters, jointly.

Results. The results show that, as in Clarida et al. (2000) and Lubik and Schorfheide (2004), monetary policy has failed to respond aggressively to inflation before Volcker. But, our estimates reveal large degrees of bounded rationality in all samples, which are disregarded in the previous literature. With the estimated extent of inattention, the best-fitting models are characterized by equilibrium determinacy both in the pre-1979 and post-1982 periods.

\footnotetext{
${ }^{1}$ Besides a transition from "bad" to "good" monetary policy, other explanations include regime changes in the volatility of shocks, i.e., changes in luck (Sims and Zha, 2006), and learning behavior by the central bank (Milani, 2008, 2014).
} 


\section{Behavioral New Keynesian Model}

We assume that the economy can be summarized by the Behavioral New Keynesian Model introduced by Gabaix (2018). The log-linearized equations are given by:

$$
\begin{aligned}
x_{t} & =M E_{t} x_{t+1}-\sigma\left(i_{t}-E_{t} \pi_{t+1}\right)+r_{t} \\
\pi_{t} & =\beta M^{f} E_{t} \pi_{t+1}+\kappa x_{t}+u_{t} \\
i_{t} & =\rho i_{t-1}+(1-\rho)\left[\chi_{\pi} \pi_{t}+\chi_{x} x_{t}\right]+\varepsilon_{t} .
\end{aligned}
$$

where $x_{t}$ denotes the output gap, $\pi_{t}$ inflation, $i_{t}$ the nominal interest rate, $r_{t}$ and $u_{t}$ are $\mathrm{AR}(1)$ demand and supply disturbances, and $\varepsilon_{t}$ is an i.i.d. monetary policy shock. The parameter $\sigma$ measures the elasticity of intertemporal substitution, $\kappa$ denotes the slope of the Phillips curve, which inversely depends on price stickiness, and $\rho, \chi_{\pi}, \chi_{x}$, are monetary policy coefficients.

The main difference between the Behavioral and the standard New Keynesian model is the addition of terms $M, M_{f} \in[0,1]$ in front of expected output gap and inflation. Gabaix (2018) derives the model as the outcome of "cognitive discounting" in the way economic agents perceive the future. When solving their infinite-horizon optimization problems, boundedlyrational consumers and firms discount more strongly events that are expected to happen further into the future. Therefore, $M$ and $M_{f}$ can represent degrees of myopia or macroeconomic inattention. $^{2}$ Gabaix shows that some degree of inattention toward the future can explain puzzles in monetary economics, such as the "forward guidance puzzle" (Del Negro et al., 2015). ${ }^{3}$

2.1. Determinacy Conditions. In the benchmark New Keynesian model, a failure of the Taylor principle triggers indeterminacy and sunspot-driven fluctuations in the economy. Bounded rationality, however, alters the conditions for indeterminacy. Partially-inattentive agents become less reactive to future expected fluctuations and, as a result, the economy becomes potentially less sensitive to instability.

As shown in Gabaix (2018), the determinacy conditions for the behavioral model become

$$
\chi_{\pi}+\left(\frac{1-\beta M_{f}}{\kappa}\right) \chi_{x}+\frac{\left(1-\beta M_{f}\right)(1-M)}{\kappa \sigma}>1
$$

\footnotetext{
${ }^{2}$ In the benchmark model, there's no discounting parameter directly in front of $E_{t} \pi_{t+1}$ in equation (1). This is the most parsimonious specification, where only the level of macro attention matters. Gabaix (2018) shows that if different degrees of attention are allowed for real interest rates and income, the corresponding inattention parameters will affect $\sigma$ : in that case, expected inflation and interest rates will also be discounted, but at the same rate. Gabaix also discusses the possibility to allow consumers to suffer from nominal illusion: in that case, $E_{t} \pi_{t+1}$ in equation (1) would be replaced by the behavioral expectation $m_{\pi} E_{t} \pi_{t+1}$, where a new discount term $m_{\pi}$ appears.

${ }^{3}$ The forward guidance puzzle implies that, under RE, monetary policy announcements that lead to changes in interest rates far into the future have unrealistically large effects on contemporaneous variables.
} 
Therefore, the cognitive discounting coefficients $M$ and $M_{f}$ expand the determinacy region. Monetary policies that respond far less than one-to-one with respect to inflation may still be conducive to determinacy, as long as the degree of bounded rationality is sufficiently large. ${ }^{4}$

\section{Bayesian Estimation under Determinacy/Indeterminacy}

We use quarterly data on output gap, inflation, and interest rates. The output gap is obtained as the log difference between real GDP and real Potential GDP, using the CBO's series; inflation is the quarterly log-difference of the GDP Implicit Price Deflator; the interest rate is given by the Federal Funds rate transformed into a quarterly rate. ${ }^{5}$

We estimate the Behavioral New Keynesian model by allowing the parameters to fall either in the determinacy or indeterminacy regions. We consider two samples: pre-Volcker, i.e., the period between 1954:Q3 (the first date of availability of FFR data) and 1979:Q3; postVolcker, i.e., the period spanning the years between 1982:Q1 and 2007:Q3 (ending before the Great Recession). ${ }^{6}$

The model under determinacy and indeterminacy is solved and estimated using the approach in Bianchi and Nicoló (2019). We estimate the model using Bayesian methods. We generate 1,250,000 draws using a Metropolis-Hastings algorithm, and discard the first 25\% as burn-in.

The priors are shown in Table 1 . We assume a $N(1,0.5)$ prior for the feedback to inflation coefficient $\chi_{\pi}$ before 1979, and $N(1.5,0.5)$ afterwards. For the inattention parameters $M$ and $M_{f}$, we choose a Beta prior with mean 0.8 and standard deviation 0.15. Later, we'll examine robustness to a different prior that more strongly favors the benchmark case of no inattention. For the sunspot shock, we assume an Inverse-Gamma prior for its standard deviation, and Uniform[-1,1] distributions for the corresponding correlation parameters.

\section{Results}

Table 1 shows the posterior estimates for the pre-1979 and post-1982 periods, both under determinacy and indeterminacy. The bottom row reports the marginal likelihoods.

The results show that the estimated monetary policy response to inflation indeed falls below 1 in the pre-1979 period: the mean estimates equal 0.6174 under indeterminacy and 0.8859 under determinacy. But, in the latter case, a failure of the central bank to move nominal interest rates more than one-to-one with respect to inflation is still conducive to determinacy, as a consequence of bounded rationality. In the determinate case, the extent of inattention is substantial, particularly so for inattention regarding inflation: $M$ has a posterior mean equal to 0.8493 and $M_{f}$ to 0.5979 . Under inattention, agents become less

\footnotetext{
${ }^{4}$ In principle, a unique determinate equilibrium can exist even if $\chi_{\pi}=\chi_{x}=0$, if bounded rationality makes the term $\frac{\left(1-\beta M_{f}\right)(1-M)}{\kappa \sigma}$ large enough.

${ }^{5}$ All data series have been obtained from FRED, the Federal Reserve Economic Database.

${ }^{6}$ As convention, the period between 1979 and 1982 is excluded, as characterized by a different monetary policy regime, based on non-borrowed reserves targeting.
} 
reactive to future decisions by others, hence reducing the strength of complementarities in the aggregate. Therefore, indeterminacy becomes less likely.

Moreover, model comparison shows that the best-fitting model for the pre-Volcker sample remains one characterized by determinacy. The log marginal likelihood is -184.34 , versus -185.18 for indeterminacy. These values imply posterior model probabilities equal to 0.7 and 0.3 , respectively. Our conclusion, therefore, contrasts with the influential findings of pre-1979 indeterminacy in Clarida et al. (2000) and Lubik and Schorfheide (2004).

While the empirical evidence favors determinacy, the model probabilities are sufficiently close that one can fairly conclude that pre-1979 data may be roughly consistent with two starkly different worlds. The first, characterized by a unique, determinate, equilibrium, modest degrees of intertemporal substitution, high price stickiness, and sizable degrees of bounded rationality. The second, characterized by indeterminacy and sunspot-driven fluctuations, in a system with higher elasticity of substitution, more flexible prices, and only minimal levels of bounded rationality. ${ }^{7}$

Figure 1 compares the dynamics of the economy under determinacy and indeterminacy. The impulse responses under determinacy are typical. Under indeterminacy, several of the responses are significantly different and change sign: inflation and interest rates respond negatively to expansionary demand shocks, rather than positively; supply shocks reduce inflation in the medium term, after the initial increase; inflation goes up in response to contractionary monetary policy shocks. Under indeterminacy, sunspots also play a role and explain $8 \%$ and $14 \%$ of the forecast-error variance of output and inflation. ${ }^{8}$

Turning to the post-1982 sample, the estimates strongly favor determinacy, as expected (the model probability jumps to 0.9999$)$. Monetary policy reacts more aggressively to inflation $\left(\chi_{\pi}=2.278\right)$. The extent of inattention to future expectations is larger than in the 1960s and 1970s, and again even more significant for inflation $\left(M=0.7059, M_{f}=0.4122\right)$.

The estimates for inattention parameters are consistent with the discussion in Gabaix (2018): the sparsity microfoundation should make agents much less reactive in a low-volatility economy (post 1982), while attention would optimally increase when volatility turns higher.

4.1. Robustness. Table 2 shows the posterior estimates for a number of robustness checks. We report and discuss only pre-1979 estimations, since there is clear evidence of determinacy in the post-1982 sample, regardless of assumptions.

4.1.1. Different priors for $M, M_{f}$ coefficients. We replace the priors for the inattention coefficients $M$ and $M_{f}$, to assign most probability mass to the special case of no inattention (the benchmark NK model). This is achieved by assuming that $1-M$ and $1-M_{f}$ follow

\footnotetext{
${ }^{7}$ In our estimation, we have allowed all parameters to vary across determinacy and indeterminacy regimes. An alternative would be to estimate the model as in Bianchi (2013), where only the monetary policy parameters and the volatilities of the shocks are allowed to be regime-dependent, according to Markov-switching processes, while the other parameters are maintained fixed across regimes.

${ }^{8}$ They explain much larger shares for inflation $(\approx 40 \%)$ in the short-run.
} 
$\Gamma(0.3,0.3)$ priors (as they imply largest probability for inattention equal to zero, and lower probability as the degree of inattention increases). Despite the priors, the estimates still favor substantial degrees of myopia: the posterior means equal 0.8224 for $M$ and 0.4281 for $M_{f}$.

4.1.2. Post-1960 Sample. We re-estimate the model starting in 1960:Q1 rather than 1954:Q3. The results are again similar, with posterior model probabilities equal to 0.78 for determinacy and 0.22 for indeterminacy.

4.1.3. Operational Taylor Rule. Contemporaneous variables may not be available in realtime to the central bank; therefore, here we allow for an operational policy rule, which responds only to lagged values. Determinacy is still preferred to indeterminacy before 1979 .

4.1.4. Survey Inflation Expectations. Inflation expectations in the model are now required to match mean inflation forecasts from the Survey of Professional Forecasters, as an additional observable. ${ }^{9}$ The data again favor determinacy with large estimates of inattention.

\section{Conclusions}

A prominent empirical result in the New Keynesian literature finds that passive monetary policy in the 1960-1970s may have been responsible for macroeconomic instability in the period. A switch to a more aggressive policy toward inflation, instead, has contributed to the reduction in volatility and the macroeconomic stability that started in the early 1980s.

Our paper shows that those results hinge on the conventional assumption of rational expectations with no role for bounded rationality or inattention by consumers and firms. We show that the data favor determinacy even in the pre-1979 sample. Monetary policy was indeed fairly passive toward inflation before Volcker, but passive policy coexisted with significant levels of bounded rationality, which prevented the economy from falling into sunspot equilibria.

\section{REFERENCES}

[1] Bianchi, F., (2013), "Regime Switches, Agents' Beliefs, and Post-World War II U.S. Macroeconomic Dynamics," The Review of Economic Studies, 80(2), 463-490.

[2] Bianchi, F., and G. Nicoló, (2019), "A Generalized Approach to Indeterminacy in Linear Rational Expectations Models", Finance and Economics Discussion Series 2019-033, Washington: Board of Governors of the Federal Reserve System.

[3] Clarida, R., Galí, J., and M. Gertler, (2000). "Monetary Policy Rules and Macroeconomic Stability: Evidence and Some Theory", The Quarterly Journal of Economics, 115(1), 147-180.

[4] Del Negro, M., Giannoni, M., and C. Patterson, (2015). "The Forward Guidance Puzzle", Staff Reports 574, Federal Reserve Bank of New York.

\footnotetext{
${ }^{9}$ We allow for an i.i.d. measurement error in the observation equation, with a prior for its standard deviation following a $\Gamma(0.15,0.15)$.
} 
[5] Gabaix, X., (2014). "A Sparsity-Based Model of Bounded Rationality," The Quarterly Journal of Economics, 129(4), 1661-1710.

[6] Gabaix, X., (2016). "Behavioral Macroeconomics Via Sparse Dynamic Programming," NBER Working Paper 21848, NBER, Inc.

[7] Gabaix, X., (2018). "A Behavioral New Keynesian Model," mimeo, Harvard University.

[8] Lubik, T.A., and F. Schorfheide, (2004). "Testing for Indeterminacy: An Application to U.S. Monetary Policy," American Economic Review, 94(1), 190-217.

[9] Milani, F., (2008). "Learning, Monetary Policy Rules, and Macroeconomic Stability," Journal of Economic Dynamics and Control, 32(10), 3148-3165.

[10] Milani, F., (2014). "Learning and Time-Varying Macroeconomic Volatility," Journal of Economic Dynamics and Control, 47(C), 94-114.

[11] Sims, C.A., and T. Zha, (2006). "Were There Regime Switches in U.S. Monetary Policy?" American Economic Review, 96(1), 54-81. 


\begin{tabular}{|c|c|c|c|c|c|}
\hline \multirow[t]{3}{*}{ Param. } & \multirow[t]{3}{*}{ Prior Distr. } & \multicolumn{4}{|c|}{ Posterior Distributions } \\
\hline & & \multicolumn{2}{|c|}{ 1954-1979 } & \multicolumn{2}{|c|}{$\underline{1982-2007}$} \\
\hline & & Indeterminacy & Determinacy & Indeterminacy & Determinacy \\
\hline$I S \& P C$ & & & & & \\
\hline$\sigma$ & $\Gamma(1.00,0.75)$ & $1.1087[0.26,1.96]$ & $0.0981[0.003,0.19]$ & $0.2361[0.03,0.44]$ & $0.0698[0.002,0.14$ \\
\hline$\kappa$ & $\Gamma(0.10,0.05)$ & $0.1449[0.01,0.25]$ & $0.0207[0.005,0.04]$ & $0.1311[0.07,0.19]$ & $0.0289[0.008,0.05$ \\
\hline Taylor Rule & & & & & \\
\hline$\rho$ & $B(0.7,0.1)$ & $0.8154[0.75,0.88]$ & $0.8034[0.75,0.86]$ & $0.9249[0.89,0.96]$ & $0.8777[0.85,0.91]$ \\
\hline$\chi_{\pi}$ & $N(1,0.5)$ & $0.6174[0.42,0.84]$ & $0.8859[0.66,1.11]$ & & \\
\hline & $N(1.5,0.5)$ & & & $0.5955[0.35,0.86]$ & $2.2780[1.81,2.76]$ \\
\hline$\chi_{x}$ & $N(0.125,0.0625)$ & $0.2005[0.13,0.27]$ & $0.1986[0.14,0.26]$ & $0.0834[0.00,0.16]$ & $0.1825[0.11,0.25]$ \\
\hline Inattention & & & & & \\
\hline$M$ & $B(0.8,0.15)$ & $0.9770[0.95,1.00]$ & $0.8493[0.68,1.00]$ & $0.9954[0.99,1.00]$ & $0.7059[0.49,0.92]$ \\
\hline$M_{f}$ & $B(0.8,0.15)$ & $0.9027[0.79,1.00]$ & $0.5979[0.35,0.85]$ & $0.5714[0.36,0.79]$ & $0.4122[0.22,0.60]$ \\
\hline Shocks & & & & & \\
\hline$\rho_{r}$ & $B(0.5,0.2)$ & $0.6851[0.40,0.93]$ & $0.8726[0.81,0.93]$ & $0.6934[0.46,0.93]$ & $0.9475[0.92,0.98]$ \\
\hline$\rho_{u}$ & $B(0.5,0.2)$ & $0.7978[0.58,0.97]$ & $0.8070[0.72,0.89]$ & $0.9496[0.92,0.98]$ & $0.9246[0.87,0.98]$ \\
\hline$\sigma_{r}$ & $\Gamma^{-1}(0.30,1)$ & $0.3626[0.10,0.62]$ & $0.2845[0.12,0.46]$ & $0.1838[0.09,0.27]$ & $0.1926[0.08,0.30]$ \\
\hline$\sigma_{u}$ & $\Gamma^{-1}(0.30,1)$ & $0.2870[0.17,0.40]$ & $0.1976[0.12,0.28]$ & $0.1180[0.08,0.16]$ & $0.1123[0.08,0.14]$ \\
\hline$\sigma_{\varepsilon}$ & $\Gamma^{-1}(0.30,1)$ & $0.1816[0.16,0.20]$ & $0.1793[0.16,0.20]$ & $0.1541[0.13,0.17]$ & $0.1429[0.13,0.16]$ \\
\hline Sunspot & & & & & \\
\hline$\sigma_{\zeta}$ & $\Gamma^{-1}(0.30,1)$ & $0.3163[0.24,0.38]$ & & $0.1900[0.16,0.22]$ & \\
\hline$\rho_{\zeta_{t}, r_{t}}$ & $U[-1,1]$ & $-0.2153[-0.86,0.36]$ & & $-0.4627[-0.81,-0.11]$ & \\
\hline$\rho_{\zeta_{t}, u_{t}}$ & $U[-1,1]$ & $0.6426[0.36,0.97]$ & & $0.6985[0.47,0.95]$ & \\
\hline$\rho_{\zeta_{t}, \varepsilon_{t}}$ & $U[-1,1]$ & $0.1093[-0.00,0.3]$ & & $0.2000[0.08,0.33]$ & \\
\hline Log-MargL & & -185.18 & -184.34 & -44 & -32.09 \\
\hline
\end{tabular}

TABle 1. Posterior Estimates. Note: The Table reports mean posterior estimates, along with $5 \%$ and $95 \%$ percentiles. We ran 1,250,000 $\mathrm{MH}$ draws, discarding the first $25 \%$ as initial burn-in. Marginal likelihoods are computed using Geweke's modified harmonic mean approximation. 


\begin{tabular}{|c|c|c|c|c|c|c|c|c|}
\hline \multirow[t]{3}{*}{ Param. } & \multicolumn{8}{|c|}{ Posterior Distributions (Pre-1979 only) } \\
\hline & \multicolumn{2}{|c|}{$\underline{\text { Diff. } M, M_{f} \text { Priors }}$} & \multicolumn{2}{|c|}{$\underline{\text { Post-1960 }}$} & \multicolumn{2}{|c|}{$\underline{\text { Lagged TR }}$} & \multicolumn{2}{|c|}{$\underline{\text { Survey Exp. }}$} \\
\hline & Indeterminacy & Determinacy & Indeterminacy & Determinacy & Indeterminacy & Determinacy & Indeterminacy & Determinacy \\
\hline$I S \& P C$ & & & & & & & & \\
\hline$\sigma$ & $0.9867[0.17,1.80]$ & $0.0992[0.003,0.20]$ & $1.0130[0.39,1.61]$ & $0.1506[0.005,0.29]$ & $0.8274[0.17,1.45]$ & $0.0620[0.001,0.13]$ & $0.7059[0.05,1.32]$ & $0.1255[0.004,0.24]$ \\
\hline$\kappa$ & $0.1267[0.01,0.24]$ & $0.0254[0.006,0.04]$ & $0.1432[0.03,0.24]$ & $0.0242[0.006,0.04]$ & $0.1317[0.03,0.22]$ & $0.0208[0.005,0.04]$ & $0.0783[0.01,0.16]$ & $0.0199[0.005,0.03]$ \\
\hline \multicolumn{9}{|l|}{ Taylor Rule } \\
\hline$\rho$ & $0.8142[0.75,0.88]$ & $0.8035[0.75,0.86]$ & $0.7280[0.63,0.82]$ & $0.7276[0.65,0.81]$ & $0.8401[0.78,0.90]$ & $0.8017[0.74,0.87]$ & $0.8274[0.77,0.89]$ & $0.8027[0.75,0.86]$ \\
\hline$\chi_{\pi}$ & $0.6196[0.41,0.83]$ & $0.8859[0.66,1.11]$ & $0.6870[0.52,0.87]$ & $0.8727[0.68,1.07]$ & $0.4836[0.24,0.73]$ & $0.8182[0.59,1.05]$ & $0.5304[0.29,0.77]$ & $0.8859[0.66,1.10]$ \\
\hline$\chi_{x}$ & $0.1999[0.13,0.27]$ & $0.1985[0.13,0.26]$ & $0.1727[0.11,0.23]$ & $0.1775[0.12,0.23]$ & $0.1732[0.10,0.24]$ & $0.1671[0.10,0.23]$ & $0.1918[0.12,0.26]$ & $0.2005[0.14,0.26]$ \\
\hline \multicolumn{9}{|l|}{ Inattention } \\
\hline & $0.9784[0.95,1.00]$ & $0.8224[0.58,1.00]$ & $0.9727[0.94,1.00]$ & $0.8535[0.68,1.00]$ & $0.9851[0.97,1.00]$ & $0.8267[0.64,1.00]$ & $0.9779[0.95,1.00]$ & $0.8504[0.68,1.00]$ \\
\hline$M_{f}$ & $0.9201[0.80,1.00]$ & $0.4281[0.03,0.75]$ & $0.9201[0.82,1.00]$ & $0.5838[0.34,0.83]$ & $0.8210[0.67,1.00]$ & $0.5965[0.35,0.85]$ & $0.9254[0.81,1.00]$ & $0.6224[0.35,0.89]$ \\
\hline \multicolumn{9}{|l|}{ Shocks } \\
\hline$\rho_{r}$ & $0.7068[0.41,0.93]$ & $0.8742[0.81,0.93]$ & $0.7371[0.46,0.95]$ & $0.8787[0.82,0.94]$ & $0.5948[0.32,0.91]$ & $0.8732[0.81,0.93]$ & $0.8041[0.66,0.95]$ & $0.8711[0.81,0.93]$ \\
\hline$\rho_{u}$ & $0.7459[0.41,0.98]$ & $0.8087[0.73,0.89]$ & $0.8445[0.71,0.97]$ & $0.8307[0.75,0.91]$ & $0.8503[0.75,0.96]$ & $0.8116[0.73,0.89]$ & $0.6871[0.44,0.93]$ & $0.6874[0.60,0.78]$ \\
\hline$\sigma_{r}$ & $0.3399[0.11,0.58]$ & $0.3072[0.11,0.54]$ & $0.3135[0.11,0.51]$ & $0.2629[0.11,0.42]$ & $0.3040[0.09,0.53]$ & $0.2972[0.12,0.48]$ & $0.3236[0.12,0.53]$ & $0.2880[0.13,0.46]$ \\
\hline$\sigma_{u}$ & $0.2810[0.17,0.39]$ & $0.2467[0.14,0.36]$ & $0.2489[0.15,0.35]$ & $0.1823[0.11,0.25]$ & $0.2531[0.14,0.36]$ & $0.1967[0.12,0.28]$ & $0.2389[0.16,0.32]$ & $0.2261[0.15,0.31]$ \\
\hline$\sigma_{\varepsilon}$ & $0.1814[0.16,0.20]$ & $0.1795[0.16,0.20]$ & $0.1889[0.16,0.21]$ & $0.1863[0.16,0.21]$ & $0.1894[0.17,0.21]$ & $0.1870[0.17,0.21]$ & $0.1831[0.16,0.20]$ & $0.1796[0.16,0.20]$ \\
\hline \multicolumn{9}{|l|}{ Sunspot } \\
\hline$\sigma_{\zeta}$ & $0.3089[0.23,0.39]$ & & $0.2881[0.22,0.35]$ & & $0.3486[0.29,0.41]$ & & $0.3529[0.27,0.44]$ & \\
\hline$\rho_{\zeta_{t}, r_{t}}$ & $-0.1829[-0.86,0.35]$ & & $-0.1177[-0.86,0.46]$ & & $-0.3336[-0.81,0.22]$ & & $-0.0316[-0.37,0.30]$ & \\
\hline$\rho_{\zeta_{t}, u_{t}}$ & $0.6756[0.37,0.98]$ & & $0.5963[0.27,0.96]$ & & $0.6563[0.45,0.92]$ & & $0.7890[0.61,0.97]$ & \\
\hline$\rho_{\zeta_{t}, \varepsilon_{t}}$ & $0.1057[-0.10,0.29]$ & & $0.0504[-0.19,0.28]$ & & $0.2066[0.05,0.36]$ & & $0.2014[0.03,0.37]$ & \\
\hline \multicolumn{9}{|l|}{ M.E. } \\
\hline$\sigma_{m e}$ & & & & & & & $0.4837[0.23,0.66]$ & $0.4822[0.39,0.57]$ \\
\hline Log-MargL & -184.07 & -183.85 & -139.24 & -137.99 & -189.27 & -189.09 & -228.96 & -221.02 \\
\hline
\end{tabular}

TABLE 2. Robustness checks. Note: The Table reports mean posterior estimates, along with $5 \%$ and $95 \%$ percentiles, for the following robustness checks: priors equal to $\Gamma(0.3,0.3)$ for $M, M_{f}$; estimation sample starting in 1960:Q1 rather than 1954:Q3; Taylor rule responding to lagged variables; addition of survey inflation expectations as an observable. 

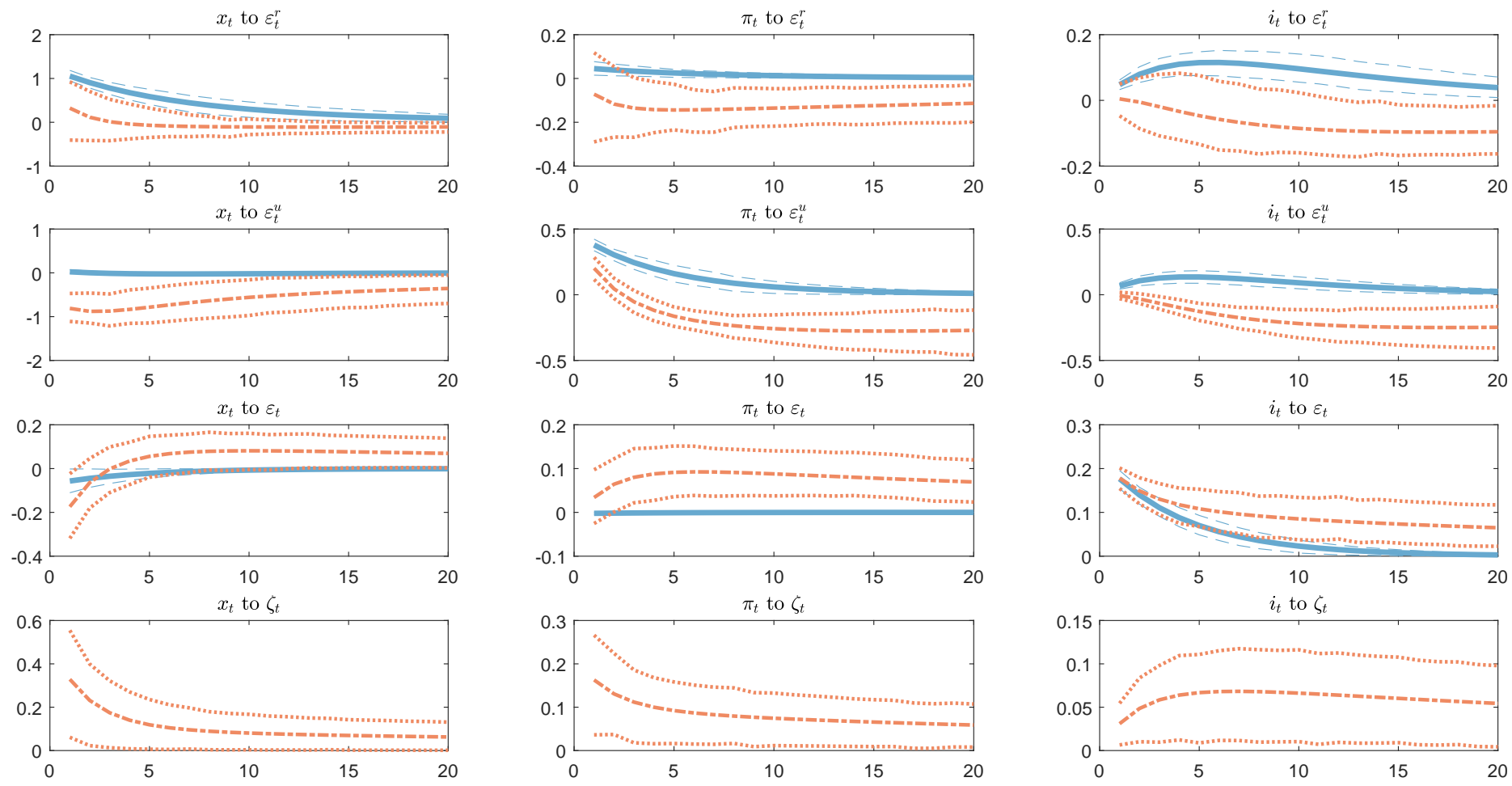

Figure 1. Impulse Response Functions under Determinacy and Indeterminacy (pre-1979). Note: The Figure shows impulse responses of output gap, inflation, and interest rates, to demand $\left(\varepsilon_{t}^{r}\right)$, cost-push $\left(\varepsilon_{t}^{u}\right)$, monetary policy $\left(\varepsilon_{t}\right)$, and sunspot shocks $\left(\zeta_{t}\right)$. Solid blue lines refer to determinacy, dash-dotted red lines to indeterminacy. The plots show mean impulse responses and 5\%-95\% bands. 